\section{The generality of the "target-pointing effect" in prism adaptation*}

\author{
ROBERT B. WELCH and MERILYN R. ABEL \\ University of Kansas, Lawrence, Kans. 66044
}

Twelve Ss were used as their own control in target-pointing and no-target conditions of prism (20 diopters) exposure. Before and after each exposure condition, target-pointing accuracy was tested via (1) forward and (2) lateral arm movement, the first of these being identical to the response made during prism exposure in the target-pointing condition. Target-pointing enhanced adaptation (the "target-pointing effect"), whether measured by the forward or lateral movement. This finding contradicts the suggestion that the target-pointing effect is limited to measures of adaptation that entail visuomotor responses identical to those occurring in the exposure period of the target-pointing condition.

In recent years visuomotor adaptation to prism-displaced vision has been the subject of intensive examination (Wohlwill, 1966). From this research some investigators (e.g., Rock, 1966) have concluded that prism adaptation is multiply determined. This notion, which has been termed the "informational discrepancy hypothesis" by Wallach (1968), states, in effect, that any form of information regarding the presence and nature of the visual rearrangement is potentially capable of inducing adaptation. One type of research lending support to this hypothesis is that in which a prism-exposure condition entailing a target is compared to a condition with no target (e.g., Coren, 1966; Freedman, 1968; Welch, 1969). In all cases it has been found that the presence of a target, and hence error-corrective feedback, leads to approximately twice the adaptation induced by a no-target condition, a phenomenon that has been called the "target-pointing effect" (Welch, in press). However, Foley \& Maynes (1969) have argued that the increase in level of adaptation that results from the provision of error-corrective feedback via target pointing may have little or no generality. These authors point out that in studies that have found enhancement of adaptation with target present, the measure of adaptation involved target pointing. Thus, the stimulus and/or response characteristics of the exposure and test periods for the target-pointing condition were quite similar to each other, while for the no-target condition they were dissimilar. The suggestion is that the target-pointing effect will be found only when the measure of adaptation involves pointing at a target. Foley \& Maynes (1969) supported this hypothesis by failing to find a difference in

*The present investigation was supported by a Eniversity of Kansas general research grant, No. 3125-5038. adaptation between target-pointing and no-target conditions when altered vision was the adaptive measure. However, still unanswered is the question of whether or not the target-pointing effect generalizes to other measures of motoric adaptation. The present study investigated this question by using as one measure of adaptation a visuomotor response quite different from that practiced during prism exposure.

\section{METHOD}

The Ss were 12 experimentally naive volunteers from an introductory psychology course at the University of Kansas.

An 11-deg leftward displacement of S's visual field was effected by means of a pair of welder's safety goggles with a 20-diopter wedge prism placed in each eyepiece. The main apparatus has been illustrated elsewhere (Welch \& Rhoades, 1969). Briefly, it consisted of a horizontal "occluding board," elevated 12 in. above a table. On S's side of the board was attached a dental impression bite plate and on E's side (201/2 in. from S's eyes) was a vertical $71 / 2 \times 3 / 16$ in. target, which hung from a tightly stretched rubber cord, running parallel to the edge of the board. The target was visible in the dark as the result of a strip of luminous tape that ran its length.

The experimental session consisted of three periods: preexposure, exposure, and postexposure, the procedure for the first and third being identical. All three parts of the experiment took place in the dark with $S$ biting into the bite plate, which served to stabilize his head position. During the preand postexposure periods, $S$ wore no goggles and was measured in two different ways on his ability to localize the luminous target, positioned directly in front of him. One manner of pointing ("forward movement") required that $S$ reach forward with his right hand in contact with the underside of the board and terminate the movement by curling his index finger around the far edge at the apparent location of the target. He then returned his hand to the near side of the board in readiness for the next response. A wooden barrier kept $S$ from noting his accuracy. Eight responses were made during the preexposure period and eight during the postexposure period. The $S$ wore a luminous rubber finger on his right index finger, with a thin wire attached to its palmar surface. On each pointing attempt the wire left a mark in a strip of clay that paralleled a ruler on the far edge of the occluding board, thus allowing for a precise measurement of his accuracy. After the mark was made, a sliding door, 7 in. from $S$ 's eyes, was lowered, $\mathbf{S}$ closed his eyes, the lights were turned on, and the response recorded. The second way in which $S$ localized the target during the pre- and postexposure periods was by means of the "lateral movement." This response began with S's right arm extended ahead of his body and resting on the table, with the hand located directly beneath the far edge of the occluding board, to the far right (on half of the trials) or to the far left. On command, $\mathrm{S}$ raised his right hand straight up until his knuckles touched the underside of the occluding board and his index finger was extended past the far edge of the board, but not curled around it. The barrier kept $S$ from seeing his finger. Next, the hand was moved laterally until $S$ judged his index finger to be in line with the target, at which point he curled his finger around the edge, leaving a mark in the clay. Eight pre-and eight postexposure measures of this sort were taken. The order of the preexposure measures was always forward movement first and lateral movement second, while the order of the

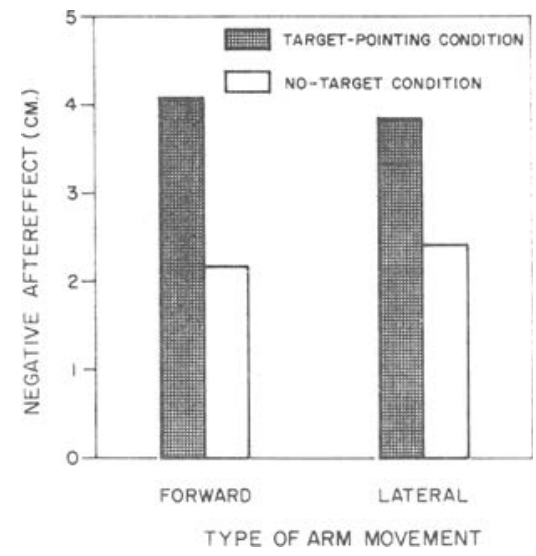

Fig. 1. Negative aftereffect (centimeters) for target-pointing and no-target conditions as measured by forward and lateral movements. 
postexposure measures was counterbalanced across $S$ s.

It was during the exposure period that $S$ wore the prism goggles and was allowed to see the displaced image of his luminous finger. The two conditions of the experiment were differentiated by the nature of the exposure period. The $S$ was used as his own control in both conditions, which were separated in time by no less than 1 week. The order of the conditions was counterbalanced across Ss, while the order of the two types of postexposure response for a given $S$ was the same for both experimental conditions. In the target-pointing condition $S$ pointed at the luminous target and, due to the removal of the barrier, was allowed to note his accuracy. He pointed in time to the recorded beat of a metronome set for one beat every $2 \mathrm{sec}$, with his finger curled around the far edge on one beat and his hand to his right, under and in contact with the near edge of the occluding board, on the next beat. This movement was identical to the forward movement of the pre- and postexposure periods. During the first five trials the target was located directly in front of $S$; for the remaining trials its position was varied in a fixed irregular order. The $S$ was instructed to correct for his error on every trial, not by moving his visible finger sideways, but by noting his error and trying to bring his finger up accurately on the next trial. In the no-target condition the target was absent during the exposure period. The $\mathrm{S}$ reached under the board in time to the metronome, but merely curled his finger around the edge of the board and viewed it for the short period (approximately $1 \mathrm{sec}$ ) that it was visible. The $S$ was asked to vary "randomly" the position in which he placed the finger, attempting to utilize the entire range of possible positions. A total of 35 responses occurred during the exposure period. There were two measures of adaptation: The pre-post difference in target-pointing accuracy when using the forward movement and the same difference when using the lateral movement. If there was a compensatory (rightward) shift in the mean target-pointing localization, this was taken to indicate that adaptation had occurred; this measure is known as the "negative aftereffect" (NA).

\section{RESULTS}

Figure 1 presents the results of the experiment. A two-tailed test for correlated means was carried out on the data for each of the two dependent variables. The tests indicated that the apparent difference in NA in favor of the target-pointing condition was significant $(p<.025)$ for both the forward-movement measure $(\mathrm{t}=2.72)$ and the lateral-movement measure $(\mathrm{t}=2.70)$. A third $t$ test of the same kind revealed that there was no difference between the two types of arm movement with regard to the amount by which target pointing augmented adaptation $(t=.71, p>.05)$.

$$
\text { DISCUSSION }
$$

It is true that the forward-movement and lateral-movement localization responses of the present study both represented ways of pointing at a target. Therefore, it might be argued that the question of whether or not the target-pointing effect generalizes to behaviors different from the one engaged in during the target-pointing exposure period was not actually tested. However, it must be understood that all measures of visuomotor adaptation involve the motoric localization of a visual object and, thus, target pointing. The real question is: Will the target-pointing effect remain if one makes the stimulus and/or response characteristics of the target-pointing measure of adaptation different from those of the target-pointing exposure period? The present study varied a response characteristic (type of arm movement) and found that the target-pointing effect was neither abolished nor attenuated as a result. Thus, it may be tentatively concluded that the provision of error-corrective feedback by means of target-pointing experience leads to an augmentation of generalized visuomotor adaptation, a finding that, in turn, supports the informational discrepancy hypothesis.

\section{REFERENCES}

COREN, S. Adaptation to prismatic displacement as a function of available information. Psychonomic Science, 1966, 4, 407-408.

FOLEY, J. E., \& MAYNES, F. J. Comparison of training methods in the production of prism adaptation. Journal of Experimental Psychology, 1969, 81, 151-155.

FREEDMAN, S. J. Perceptual compensation and learning. In S. J. Freedman (Ed.), The neuropsychology of spatially oriented behavior. Homewood, Ill: The Dorsey Press, 1968. Pp. 63-76.

ROCK, I. The nature of perceptual adaptation. New York: Basic Books, 1966.

WALLACH, $\mathrm{H}$. Informational discrepancy as a basis of perceptual adaptation. In $S$. $J$. Freedman (Ed.), The neuropsychology of spatially oriented behavior. Homewood, 111: The Dorsey Press, 1968. Pp. 209-229.

WELCH, R. B. Adaptation to prism-displaced vision: The importance of target-pointing. Perception \& Psychophysics, 1969, 5, 305-309.

WELCH, R. B. Prism adaptation: The 'target-pointing effect' as a function of exposure trials. Perception \& Psychophysics, in press.

WELCH, R. B., \& RHOADES, R. W. The manipulation of informational feedback and its effects upon prism adaptation. Canadian Journal of Psychology, 1969, 23, 415-428.

WOHLWILL, J. F. Perceptual learning. Annual Review of Psychology, 1966, 17, 201-232.

\title{
A reversed Poggendorff illusion
}

\author{
A. W. PRESSEY* and M. J. DEWAR \\ University of Manitoba, Winnipeg, Canada
}

The Poggendorff configuration was modified in such a manner that the direction of illusion was reversed. The reversed illusion was found to be prevalent and reliable in a sample of naive Os. It was also inversely correlated with the classical Poggendorff illusion.

If two parts of a single oblique line are interrupted by two separate parallel lines, the two obliques do not appear collinear. Such a configuration is known as the Poggendorff illusion and is illustrated in Fig. 1A. Recently, Pressey \& Sweeney (1969) have shown that both parallel lines are not necessary for the illusion to occur. In Fig. 1B, one parallel line and one

*Present address: Department of Psychology, University of Manitoba, Winnipeg 19, Canada. oblique are removed and the illusion still occurs. The oblique on the left appears to point above the dot on the right parallel line. While experimenting with Target $1 \mathrm{~B}$, one of the authors (A.W.P.) constructed the pattern shown in Fig. IC. It was then noted that a striking reversal of the classical Poggendorff illusion occurred. The oblique line on the left now appeared to point below the point of objective continuation. The present investigation was initiated to explore further the reversal phenomenon. Specifically, the problems 